\title{
Resolving the Equal Number Density Puzzle: Molecular Picture from Simulations of $\mathrm{LiCl}(\mathrm{aq})$ and $\mathrm{NaCl}(\mathrm{aq})$
}

\author{
Man Nguyen Thi Hong, ${ }^{\dagger}, \ddagger$ Ondřej Ticháček, ${ }^{\dagger} \ddagger$ Hector Martinez-Seara, ${ }^{*, \dagger}$ \\ Philip E Mason, ${ }^{\dagger}$ and Pavel Jungwirth ${ }^{* \dagger}$ \\ $\dagger$ Institute of Organic Chemistry and Biochemistry of the Czech Academy of Sciences, \\ Flemingovo nám. 542/2, 16000 Prague 6, Czech Republic \\ $\ddagger$ Contributed equally to this work \\ E-mail: hseara@gmail.com; pavel.jungwirth@uochb.cas.cz
}

\begin{abstract}
The change in number densities of aqueous solutions of alkali chlorides should be qualitatively predictable. Typically, as cations get bigger the number density of the solution decreases. However, aqueous solutions of lithium and sodium chloride exhibit at ambient conditions practically identical number densities at equal molalities despite different ionic sizes. Here, we provide an atomistic interpretation of this experimentally observed anomalous behavior using molecular dynamics simulations. The obtained results show that rigidity of the $\mathrm{Li}^{+}$first and second solvation shells and associated compromised hydrogen bonding result in practically equal average water densities in the local hydration regions for $\mathrm{Li}^{+}$and $\mathrm{Na}^{+}$ despite different sizes of the cations. In addition, in more distant regions from the cations, the water densities of these two solutions also coincide. These findings thus provide an atomistic interpretation for matching number densities of $\mathrm{LiCl}$ and $\mathrm{NaCl}$ solutions. In contrast, the number density differences between $\mathrm{NaCl}$ and $\mathrm{KCl}$ solutions, as well as between $\mathrm{LiCl}$ and $\mathrm{KCl}$ solutions behave in a regular fashion with lower number densities of solutions observed for larger cations.
\end{abstract}

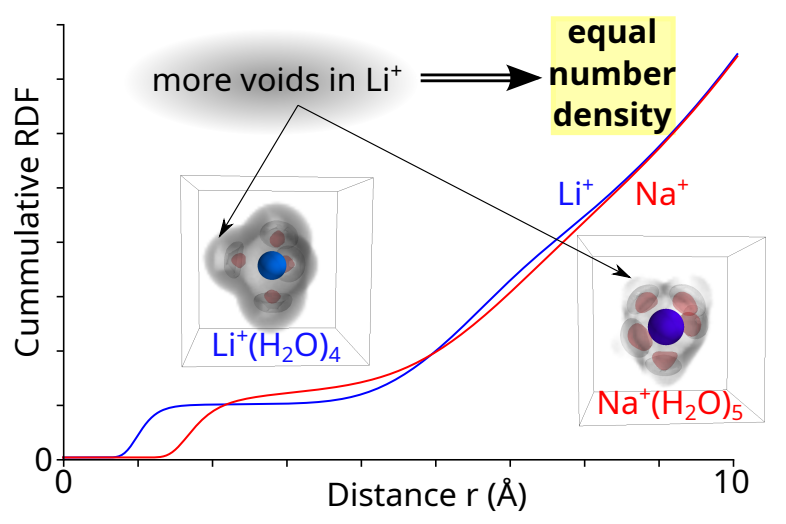




\section{Introduction}

Ions in aqueous solutions have attracted great interest due to their importance in chemistry, biology, and environmental processes ${ }^{1,2}$. For decades, a large number of investigations have been carried out to understand the behavior of ions in aqueous environments at a molecular level. Among these, a significant effort has been devoted to obtaining a comprehensive knowledge of molecular structures of hydration shells of ions using experimental and computational tools $^{3}$. This is due to the fact that macroscopic properties of salt solutions, for example viscosity or boiling and freezing points, are significantly affected by the details concerning hydration of ions ${ }^{1,3}$. The interactions between ions and water molecules have been studied using a variety of experimental methods, such as X-ray scattering measurement ${ }^{4,5}$, X-ray absorption ${ }^{6,7}$, neutron diffraction ${ }^{8,9}$, and IR spectroscopy ${ }^{10}$. From the theoretical point of view, a large number of computational approaches, such as force field and ab initio molecular dynamics ${ }^{11-14}$, mixed quantum mechanics/molecular mechanics simulations ${ }^{15}$, and quantum chemistry minimizations ${ }^{16}$ have proven to be powerful tools which significantly enhance our understanding of the properties of ionic aqueous solutions. These experimental and computational data have been used to explain how individual ions influence the hydrogen-bond network and dynamics of surrounding waters in the first few hydration shells. Accordingly, hydration of ions in bulk water has also been rationalized in terms of various aspects such as geometries of ionic hydrated structures ${ }^{11,12,17}$ and thermodynamic properties $^{13}$.

In our research, the number density which characterizes the number of atoms in solution accommodated in a unit of volume proved to be a useful property for interpretation of neutron scattering measurements ${ }^{18}$. Density is typically given in units of mass per unit volume. However, for a variety of reasons in neutron scattering a more commonly used unit is atoms per unit volume. The two density metrics focus on different physical aspects of matter and are each useful in their own right. The high number density of water is due to the fact that it is composed of small molecules being closely and tightly held together by hydrogen bonds. This means that adding ions to the solution always decreases the number density. Typically, the larger the ion, the larger the decrease. However, there is an exception to this rule, namely, equally concentrated aqueous solutions of lithium and sodium chloride have almost identical number densities at ambient conditions despite their different ionic radii ${ }^{19}$. To the best of our knowledge, this unusual and unexpected behavior of these solutions has not been explained yet. In the present work, we aim at clarifying the origin of this phenomenon and provide an atomistic interpretation thereof based on molecular dynamics simulations of concentrated aqueous solutions of $\mathrm{LiCl}, \mathrm{NaCl}$, and $\mathrm{KCl}$ (where $\mathrm{KCl}(\mathrm{aq})$ is used as reference).

\section{Methods}

\section{MD simulations}

In the present study, a set of molecular dynamics simulations was performed for three aqueous electrolytes $\mathrm{LiCl}, \mathrm{NaCl}$, and $\mathrm{KCl}$. The simulation box contained 187 ion pairs and 2598 water molecules described by the simple point charge/extended (SPC/E) model ${ }^{20}$. This means that the concentration was $4 \mathrm{~m}$, for which there are high quality of neutron scattering data ${ }^{19,21}$. The parameterization of ions in this work is based on the electronic continuum correction (ECC) approach which results in improvements in description of aqueous salt solutions due to the mean-field inclusion of electronic polarization effects ${ }^{22-24}$. Accordingly, the scaled charges and Lennard--Jones parameters applied for the studied ions come from our previous publications ${ }^{19,25}$. All the force field parameters for $\mathrm{Li}^{+}, \mathrm{Na}^{+}, \mathrm{K}^{+}$, and $\mathrm{Cl}^{-}$are listed in Table 1.

The van der Waals interactions were cut off at the distance of $1.2 \mathrm{~nm}$ and were shifted such that they vanish at the cut off distance. The long-range electrostatic interactions were accounted for by the particle-mesh Ewald al- 
Table 1: Force field parameters of the studied ions. $r_{\mathrm{i}}$ is the crystal ionic ra$\operatorname{dius}^{26}$ for the most likely coordination number. $\sigma$ and $\varepsilon$ are the van der Waals parameters. And $q$ is the atomic charge in the simulation.

\begin{tabular}{cccccr}
\hline Ion & $\begin{array}{c}r_{\mathrm{i}} \\
(\mathrm{nm})\end{array}$ & $\begin{array}{c}\sigma \\
(\mathrm{nm})\end{array}$ & $\begin{array}{c}\varepsilon \\
(\mathrm{kJ} / \mathrm{mol})\end{array}$ & $\begin{array}{c}q \\
(\mathrm{e})\end{array}$ & Ref. \\
\hline $\mathrm{Li}^{+}$ & 0.059 & 0.1800 & 0.0765 & 0.75 & 19 \\
$\mathrm{Na}^{+}$ & 0.100 & 0.2115 & 0.5443 & 0.75 & 19 \\
$\mathrm{~K}^{+}$ & 0.138 & 0.3154 & 0.4187 & 0.75 & 25 \\
$\mathrm{Cl}^{-}$ & 0.181 & 0.3782 & 0.4184 & -0.75 & 19 \\
\hline
\end{tabular}

gorithm with a real space cut off of $1.2 \mathrm{~nm}^{27}$. The Verlet list was used to speed up the neighbor search. Temperature was kept at $295 \mathrm{~K}$ by the Nose-Hoover thermostat ${ }^{28,29}$ with a coupling time of 0.5 ps. The pressure was controlled by the Parrinello-Rahman barostat ${ }^{30}$ at 1 bar with a coupling constant of 5 ps. For water molecules, the Settle algorithm was used to constrain bonds and angles ${ }^{31}$. After minimization and 100 ps of constant volume (NVT) equilibration, $2 \mu$ s of constant pressure (NpT) production simulations were performed for all three alkali halide salts with the timestep of $2 \mathrm{fs}$ and data saving every 5 ps. All molecular dynamics simulations were carried out using the Gromacs 2018.2 program package ${ }^{32}$.

\section{Alignment and clustering}

Data from simulations were analysed with the help of the MDAnalysis python package and an in-house developed software for unbiased alignment (rotation and translation) of the solvent neighborhood of each of the cations. The neighborhood was constructed as a sphere with a radius of $10 \AA$ with the cation fixed in the center. Apart from that, no other atom was fixed in the alignment procedure in order to reproduce the natural thermal noise and resulting atomic positional volume. Each cation in each saved frame of the MD simulation was analysed accounting for a total of about 50 million independent neighborhoods for each analyzed cation, i.e., $\mathrm{Li}^{+}, \mathrm{Na}^{+}$, and $\mathrm{K}^{+}$.

The aligned neighborhoods were clustered and separated into groups depending on the configuration in the first solvation shell, i.e., considering the number of oxygens and chloride anions within this shell and the configuration geometry. Note that generally a configuration is not uniquely defined by the number and type of atoms in the shell - for example, in the case of octahedral geometry with four oxygens and two chlorides, two configurations can be distinguished, one with the chlorides at opposite sides of the central cation, and the other with chlorides next to each other.

Data from each group were overlaid and the three-dimensional occupational probability density was estimated via the histogram estimate. The densities were analysed qualitatively via contour plotting and also quantitatively using an in-house method described below. In the contour plots, each domain depicts the most likely position of a single atom. The size of this domain then corresponds to the magnitude of the thermal motion of the atom. This tree-dimensional density visualisation was performed using an in-house developed software employing the python Mayavi library ${ }^{33}$.

\section{Density analysis}

In this paper, we use a novel method of quantitative analysis of the average density of a local neighborhood of an atom. The method is based on a function that we refer to as the "radially and density decomposed distribution" (r3d), which is reminiscent of the commonly used radial distribution function (RDF) but provides further spatial details crucial for interpretation of the present results. Let us briefly introduce the r3d through the following example.

Consider two different systems consisting of a salt dissolved in water. For graphical simplicity, we assume in these hypothetical examples a two dimensional coordination of the water around the salt, but the lesson learned is equally applicable to the real three dimensional case. In the first system, water molecules around the central cation are practically constrained in a well defined terta-coordinated structure, denoted as 
organised structure

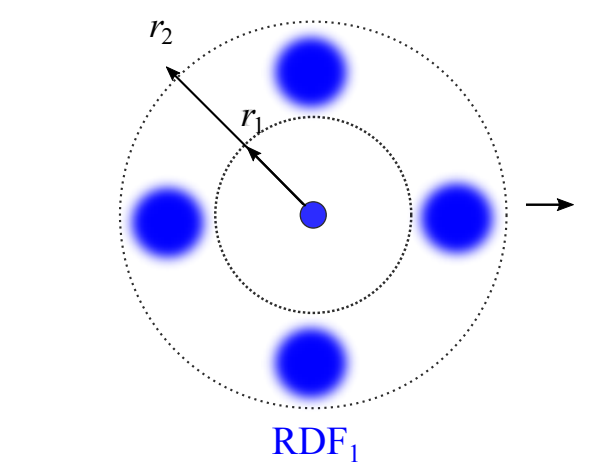

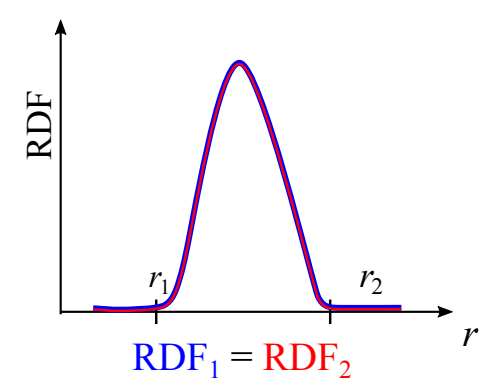

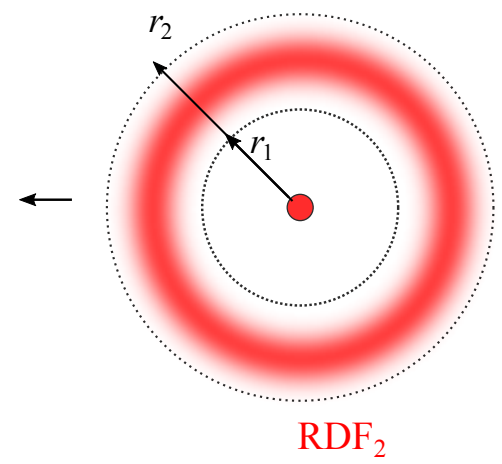

Radially-decomposed density distribution functions (r3d)
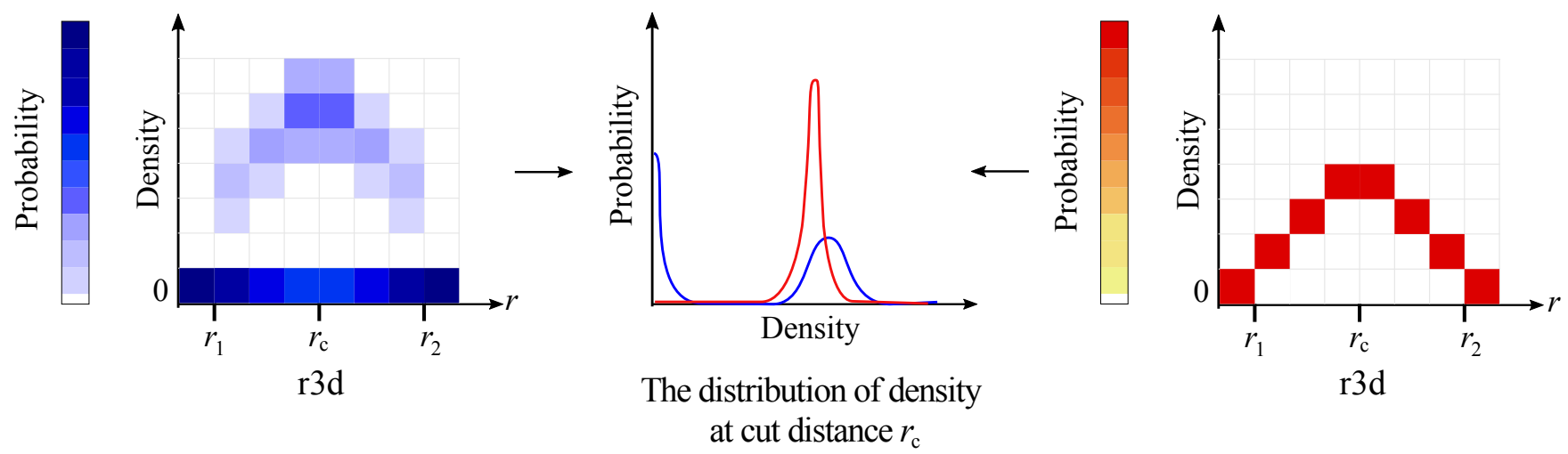

Figure 1: Illustrative comparison of the radial distribution function (RDF) and radially and density decomposed distribution (r3d) for an organised and an unorganised system.

"organized structure" in Figure 1. In the second system, denoted as "unorganized structure" in Figure 1, the same number of water molecules is radially constrained to the $2 \mathrm{~d}$ solvation shell, but the molecules move freely within that solvation shell. The water oxygen radial distribution function around the central cation of these two limiting model cases are identical, while their structures are clearly not (see Figure 1). This simple example demonstrates how insufficient RDF (which integrates over angular coordinates) can be for describing the average microscopic structure of the local solvent neighborhood.

We circumvented this drawback of RDF by introducing a "radially and density decomposed distribution" (r3d) that captures the density variability within the angular dimension as a function of radial distance. Compared to RDF, r3d contains additional information about how structured the solvent neighborhood of the cen- tral ion is in terms of higher or lower solvent density regions at a given radial distance.

The $\operatorname{r} 3 \mathrm{~d}(r, \varrho)$ is a probability function of two variables, radial distance $(r)$ and density $(\varrho)$ (either number density or physical density). It is defined as the probability to find a given value of local density $\varrho$ at a distance $r$. The value of the r3d function can be calculated from the three-dimensional density distribution function after proper alignment of the motif of interest. In practice we estimate it numerically through a histogram estimate, which is normalized (proportionally to $1 / r^{2}$ ) in the radial coordinate analogously to the normalization of RDF. The r3d function is naturally plotted as a two-dimensional heat map of $\varrho$ vs $r$ with the color scale representing the probability value.

An illustration of $\mathrm{r} 3 \mathrm{~d}(r, \varrho)$ of the two discussed limiting cases systems is presented at the bottom of Figure 1. In blue we show the "organized" system while the system with a ra- 
dially unorganised solvation shell is colored in red. Comparing these two cases we see that a structured environment display two or more regions with distinct densities $\varrho$ at a same distance $r$. In contrast, a homogeneous environment results in only one well defined region at a given $r$. We show clearly these distinctive features in the "cut" of the r3d at the distance $r_{c}$ in the lower-central panel of Figure 1.

The solvent neighborhoods of the studied cations $\left(\mathrm{Li}^{+}, \mathrm{Na}^{+}, \mathrm{K}^{+}\right)$is neither fully homogeneous nor perfectly-structured. The real situation is somewhere between these two extremes due to thermal motions, specific interactions between surrounding molecules, spatial constrains, non unique coordination numbers, as well as the quality of the alignment. To describe such more realistic scenarios it is convenient to focus on the density $\varrho$ probability distribution at a fixed distance $r$ in the $\mathrm{r} 3 \mathrm{~d}$. In mathematical terms this is the conditional $\operatorname{r3d}(\varrho \mid r)$ that at a given distance $r$ gives the probability of finding a local density value of $\varrho$. These functions can also be viewed as a "cuts" of the r3d. Note that distributions with a large variance (or a multi-modal distribution) for a given $r$ suggest a structured environment, while a uni-modal distribution with a lower variance is a sign of a mostly homogeneous environment.

A common case pertinent to this work is a multi-modal distribution with a high peak at very low density values, which is caused by the presence of "voids" in the solvent structure. Note that in this work a "void" only refers to a region defined in the aligned neighborhood of the studied cations that is mostly empty on average, i.e., empty with a high probability. Moreover, since we analyze densities of specific atoms (e.g., water oxygens), these voids can sometimes be produced simply by the respective space being mostly occupied by a different species (e.g., chloride anions).

\section{Results and Discussion}

Multiple analysis steps were required to gain a detailed insight in the structure of the solvation shells of the studied aqueous alkali chloride so- lutions. According to the steps performed, this section is organized as follows.

1. We first verified that the simulation results are in agreement with the experimental values regarding the number densities of the solutions. We also characterized these solutions using the cation-oxygen radial distribution function (RDF), and the corresponding cumulative radial distribution function (CRDF). Together they prove that the experimental number density anomaly of $\mathrm{LiCl}$ solution is well reproduced by our simulation models.

2. We characterized the first coordination shell of the cations in the studied solutions and evaluated the coordination number distribution around $\mathrm{Li}^{+}, \mathrm{Na}^{+}$, and $\mathrm{K}^{+}$. For the two most abundant configurations around each cation, we also resolved their structure in detail. Furthermore, we provided a novel visualization strategy that clearly identified regions occupied by given atoms, as well as exclusion zones, yielding realistic volumetric ratios.

3. Mean three-dimensional occupational (number) densities within the solvent neighborhood of investigated alkali cations was further analysed. For this we introduced a new function the "radially and density decomposed distribution" function (r3d), see Methods for details. This function allows to compare systematically and quantitatively $\mathrm{LiCl}, \mathrm{NaCl}$, and $\mathrm{KCl}$ solutions structures, pinpointing the source of the anomalous densities the $\mathrm{LiCl}$ and $\mathrm{NaCl}$ solutions.

4. Finally, the hydrogen bonds within the first and second hydration shells of the cations were analysed in order to rationalize at a molecular level the source of the distinct structure environment.

\section{Cation-oxygen radial distribu- tion functions}

From the present simulations, the calculated number densities of $4 \mathrm{~m}$ aqueous solutions of $\mathrm{LiCl}, \mathrm{NaCl}$, and $\mathrm{KCl}$ are 0.0971, 0.0965, and 0.0916 atoms $/ \AA^{3}$, respectively. Thus, the simulations reproduce well the experimental values which are 0.0976 for $\mathrm{LiCl}, 0.0975$ for $\mathrm{NaCl}^{19}$, and 0.0936 for $\mathrm{KCl}^{21}$. Clearly, the results show 
that the number density value of the $\mathrm{KCl}$ solution is the lowest compared with the $\mathrm{NaCl}$ and $\mathrm{LiCl}$ solutions which is in line with the largest ionic radius of potassium. The values of the ionic radii and the most abundant coordination numbers $(\mathrm{CN})$ are $0.59 \AA$ for $\mathrm{Li}^{+}\left(\mathrm{CN}_{\mathrm{Li}^{+}}=4\right)$, $1.00 \AA$ for $\mathrm{Na}^{+}\left(\mathrm{CN}_{\mathrm{Na}^{+}}=5\right)$, and $1.38 \AA$ for $\mathrm{K}^{+}$ $\left(\mathrm{CN}_{\mathrm{K}^{+}}=6\right)^{26}$. However, the fact that the number density of the $\mathrm{NaCl}$ solution is practically equivalent to that of the $\mathrm{LiCl}$ solution, although the size of the $\mathrm{Na}^{+}$ion is significantly larger than that of the $\mathrm{Li}^{+}$ion, is counter-intuitive.

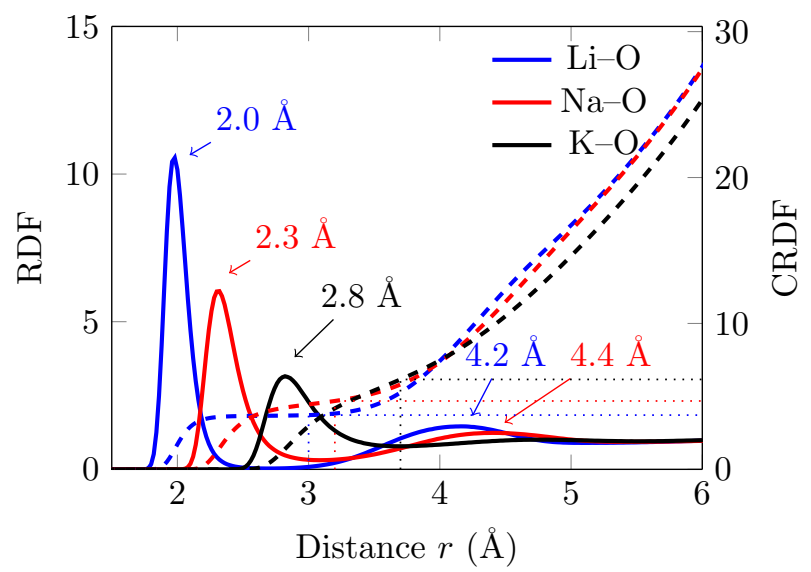

Figure 2: The alkali cation-water oxygen radial distribution functions (RDF) (solid) and the corresponding cumulative coordination numbers (CRDF) (dashed) for $4 \mathrm{~m}$ aqueous solutions of $\mathrm{LiCl}, \mathrm{NaCl}$, and $\mathrm{KCl}$. For each cation the water coordination number at the first minima is shown (dotted): $\mathrm{CN}_{\mathrm{Li}^{+}} \approx 4, \mathrm{CN}_{\mathrm{Na}^{+}} \approx 5$, and $\mathrm{CN}_{\mathrm{K}^{+}} \approx 6$.

Figure 2 displays the radial distribution functions (RDFs) of water oxygen atoms around each of the studied cations and the corresponding cumulative RDFs. The cation-oxygen RDF curves for $\mathrm{Li}^{+}$and $\mathrm{Na}^{+}$solutions exhibit each two peaks. The first peaks in $\mathrm{Li}-\mathrm{O}$ and $\mathrm{Na}-$ O RDFs are located at 2.0 and $2.3 \AA$, respectively, with the former peak being sharper than the latter. These results are in good agreement with previous literature values reported on the basis of X-ray diffraction, neutron diffraction, and molecular dynamics simulations ${ }^{7,34}$. The broader second peaks are centered at 4.2 and $4.4 \AA$ for $\mathrm{Li}^{+}$and $\mathrm{Na}^{+}$, respectively. The second hydration shell of $\mathrm{Li}^{+}$is well separated from the first one with a rather wide depleted zone in the range between 2.4 to $3.0 \AA$, which is consistent with a previous report ${ }^{35}$. For $\mathrm{NaCl}$ solution, the RDF maintains somewhat depleted density in this region. Compared to $\mathrm{Li}^{+}$and $\mathrm{Na}^{+}$hydration, $\mathrm{K}^{+}$with the largest radius exhibits a much broader first peak at around $2.8 \AA$, which is consistent with the results of ab initio molecular dynamics ${ }^{36}$ as well as X-ray and neutron diffraction experiments ${ }^{37,38}$, with the second peak and the depletion zone after the first peak being practically non-existent.

Integration of the RDFs to a given radial distance $r$ from the central cation yields the corresponding cumulative RDFs, i.e., CRDF in Fig. 2). As can be seen from Fig. 2, the average number of oxygen atoms occupying the closest coordination volume around lithium ion is 4.0, i.e., there are four water molecules coordinated to the central $\mathrm{Li}^{+}$ion in the first shell. This coordination number has been observed in a number of previous studies ${ }^{3,39,40}$. Unlike hydrated $\mathrm{Li}^{+}$, the average first shell coordination numbers for $\mathrm{Na}^{+}$and $\mathrm{K}^{+}$are not sharp integer values. For the $\mathrm{Na}^{+}$ion, there are approximately five water molecules occupying the first layer at around a cut-off of $3.2 \AA$. For the $\mathrm{K}^{+}$ion, the hydration number is about six, corresponding to an integration limit of $3.7 \AA$. Very similar coordination numbers have been reported for both cations from neutron diffraction ${ }^{41,42}$. Interestingly, for $\mathrm{Li}^{+}$and $\mathrm{Na}^{+}$at the end of the second shell, i.e., $5 \AA$ and onward the CRDFs $N_{\mathrm{Li}-\mathrm{O}}(r)$ and $N_{\mathrm{Na}-\mathrm{O}}(r)$ are practically identical. In contrast, no such concurrence takes place for the $N_{\mathrm{K}-\mathrm{O}}(r)$ curve and, consequently, the $\mathrm{KCl}$ solution has a distinctly different number density from the other two solutions. An analogous behavior is also found for the other atomic pairs, i.e., $\mathrm{Li} / \mathrm{Na} / \mathrm{K}-\mathrm{Cl}$ and $\mathrm{Li} / \mathrm{Na} / \mathrm{K}-\mathrm{H}$, as shown in Figures S1 and S2 in the Supporting Information (SI).

\section{Microscopic structures of the solutions}

Clustering of $\sim 50$ million aligned solvent neighborhoods of the cations allows for each system to separate the configurations accord- 
ing to the first hydration shell geometry and coordination number. Figure 3 shows the distribution of the configurations for each cation. The plot shows that the larger the cation the more its lowest possible coordination number increases, namely yielding $4\left(\mathrm{Li}^{+}\right), 5\left(\mathrm{Na}^{+}\right)$, and $6\left(\mathrm{~K}^{+}\right)$. The plot also shows that the most common coordination number is the lowest accessible. It is also evident that the larger the cation the more significantly are larger number of coordination numbers populated. To account at least for $90 \%$ of the configurations for lithium we only need the coordination number 4 , while for sodium we need 5 and 6 , and for potassium we need to include coordination numbers from 6 to 9 . Below, we investigate in detail specific differences between $\mathrm{Li}^{+}, \mathrm{Na}^{+}$and $\mathrm{K}^{+}$containing solutions.

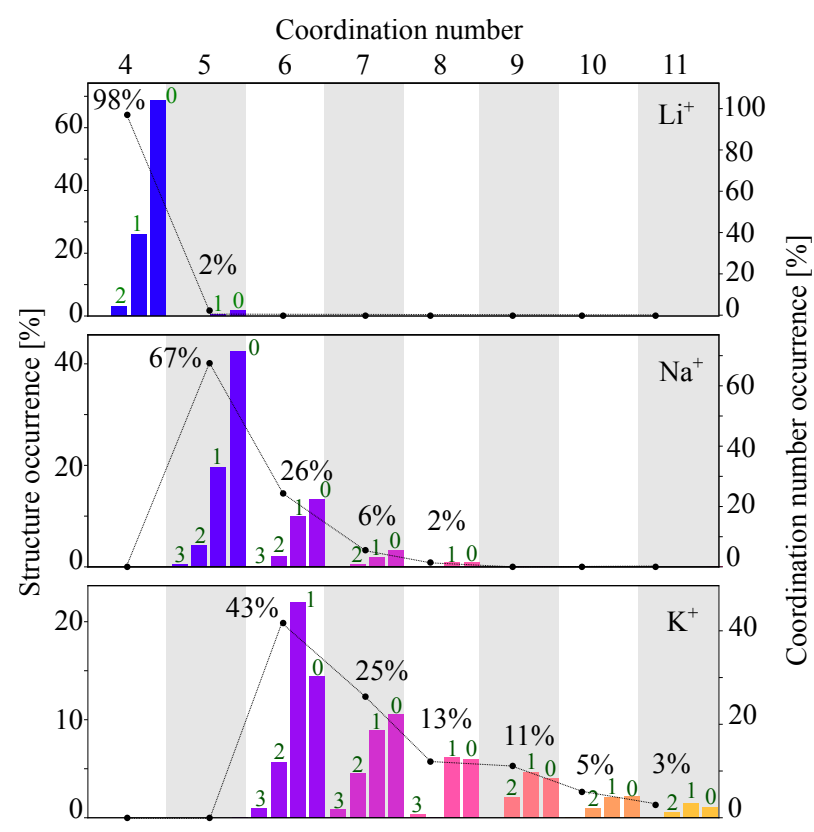

Figure 3: The percentages of individual hydration structures existing in $4 \mathrm{~m}$ aqueous solutions of $\mathrm{LiCl}, \mathrm{NaCl}$ and $\mathrm{KCl}$. Each bar within a coordination number represents a different chemical configuration. The numbers on top the bar show the number of $\mathrm{Cl}^{-}$anions in the configuration.

\section{LiCl solution}

The dominant structures of the $\mathrm{Li}^{+}$first solvation shell obtained from the MD simulation of the $4 \mathrm{~m} \mathrm{LiCl}$ aqueous solution are depicted in Figure 4. The probability distribution of the individual coordination numbers demonstrates that tetra-coordination is predominant by approximately $98 \%$ in comparison to a $2 \%$ abundance of a five-coordinated structures, see Figure 3. We see that in addition to the most abundant $\mathrm{Li}^{+}\left(\mathrm{H}_{2} \mathrm{O}\right)_{4}$ structure $(69 \%)$, the first coordination shell reports significant $\mathrm{Li}^{+}-\mathrm{Cl}^{-}$ ion paring, resulting in $26 \% \mathrm{Li}^{+}\left(\mathrm{H}_{2} \mathrm{O}\right)_{3} \mathrm{Cl}^{-}$ and $<2 \% \mathrm{Li}^{+}\left(\mathrm{H}_{2} \mathrm{O}\right)_{2}\left(\mathrm{Cl}^{-}\right)_{2}$ abundance. The average structures of the two most abundant configurations are visualized in Figure $4 \mathrm{~A}$ and 4B. Higher-order coordination are rather infrequent. Only very few five-coordinated geometries have been found including configurations of $\mathrm{Li}^{+}\left(\mathrm{H}_{2} \mathrm{O}\right)_{5}$ and $\mathrm{Li}^{+}\left(\mathrm{H}_{2} \mathrm{O}\right)_{4} \mathrm{Cl}^{-}$.

It is clear from figure $4 \mathrm{~A}$ that $\mathrm{Li}^{+}$has tetrahedral water coordination, as also reported in the earlier literature ${ }^{43-45}$. $\mathrm{As} \mathrm{Cl}^{-}$gets in direct contact with $\mathrm{Li}^{+}$, qualitatively, the tetrahedral geometry is preserved via replacing one water molecule with a chloride ion in the first shell (see Fig. 4B).

Next to the configuration structures, the voids present in the hydration shell are also plotted (Figs. 4C, D), with the spheres representing the positions of water oxygens in the first shell. It is clear from these figures that there exist well structured empty regions in the first hydration shell of $\mathrm{Li}^{+}$between the first and second shells, which is consistent with the clear separation between these shells as seen in the RDF (Figure 2).

\section{$\mathrm{NaCl}$ solution}

The first hydration shell of $\mathrm{Na}^{+}$in the $4 \mathrm{~m} \mathrm{NaCl}$ solution exhibits mainly coordination numbers of five and six with corresponding probabilities of $69 \%$ and $26 \%$ (see Figure 3). $\mathrm{Na}^{+}$shell can also achieve sevenor even eight-coordinated structures, however, with only small probabilities of $6 \%$ and $2 \%$, respectively. In stark contrast to $\mathrm{Li}^{+}$, the percentage of four-coordinated structures is negligible. The most abundant structures, i.e., $\mathrm{Na}^{+}\left(\mathrm{H}_{2} \mathrm{O}\right)_{5}(43 \%)$ and $\mathrm{Na}^{+}\left(\mathrm{H}_{2} \mathrm{O}\right)_{4} \mathrm{Cl}^{-}(21 \%)$ are penta-coordinated and are depicted in Fig- 
A

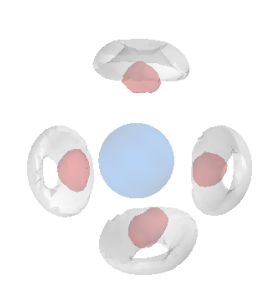

B

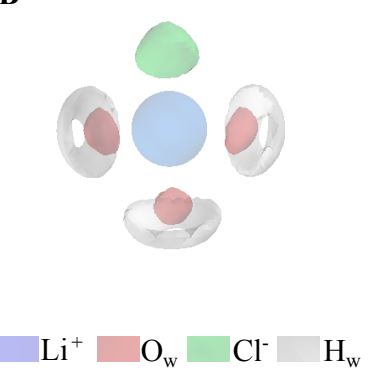

Figure 4: Structure of the two most populated configurations of the first coordination shell for $\mathrm{Li}^{+}$. The left columns show density plots of waters or chloride molecules around a central $\mathrm{Li}^{+}$. The right column depicts the voids in the same structures, with spheres showing the positions of atoms for reference. The more intense the color, the lower probability of finding oxygen atoms in corresponding region. Panels $\mathbf{A}$ and C correspond to $\mathrm{Li}^{+}\left(\mathrm{H}_{2} \mathrm{O}\right)_{4}$. Panels $\mathbf{B}$ and D are respective to $\mathrm{Li}^{+}\left(\mathrm{H}_{2} \mathrm{O}\right)_{3} \mathrm{Cl}^{-}$.

ures 5A and 5B. From all other possible configurations only two more has more than $5 \%$ abundance being the hexa-coordinated structures $\mathrm{Na}^{+}\left(\mathrm{H}_{2} \mathrm{O}\right)_{6}(12 \%)$ and $\mathrm{Na}^{+}\left(\mathrm{H}_{2} \mathrm{O}\right)_{5} \mathrm{Cl}^{-}$ $(11 \%)$ which are depicted in Figure S3 in SI.

Comparing to $\mathrm{Li}^{+}$, it is clear that the most abundant arrangements of solvent around $\mathrm{Na}^{+}$ leave fewer empty spaces in the first coordination shell, as can be seen in Figures 5C and 5D.

\section{$\mathrm{KCl}$ solution}

The larger size of the $\mathrm{K}^{+}$ion opens up the possibility of higher first shell coordination numbers. As shown in Figure 3, the broader probability distribution coordination numbers calculated from the simulated trajectory illustrates the existence of different configurations of $\mathrm{K}^{+}$in the $4 \mathrm{~m} \mathrm{KCl}$ solution with coordination numbers up to 11 . The coordination number of 6 accounts for the largest popula-
A

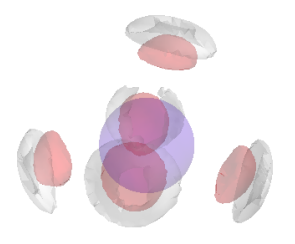

C

B

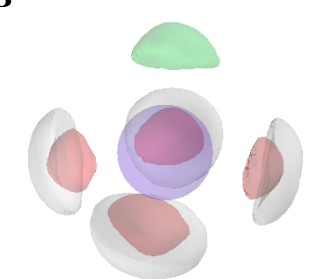

D

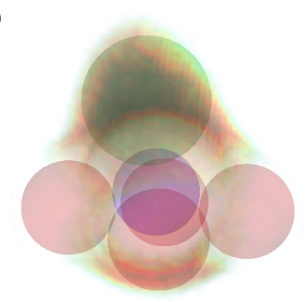

$\begin{array}{llll}\mathrm{Na}^{+} & \mathrm{O}_{\mathrm{w}} & \mathrm{Cl}^{-} & \mathrm{H}_{\mathrm{w}}\end{array}$

Figure 5: Structures of the two most populated configurations of the first coordination shell for $\mathrm{Na}^{+}$. The left column shows density plots of waters or chloride molecules around a central $\mathrm{Na}^{+}$. The right column depicts the voids in the same structures, with spheres showing the positions of atoms for reference. The more intense the color, the lower probability of finding oxygen atoms in corresponding region. Panels $\mathbf{A}$ and $\mathbf{C}$ correspond to $\mathrm{Na}^{+}\left(\mathrm{H}_{2} \mathrm{O}\right)_{5}$. Panels $\mathbf{B}$ and $\mathbf{D}$ are respective to $\mathrm{Na}^{+}\left(\mathrm{H}_{2} \mathrm{O}\right)_{4} \mathrm{Cl}^{-}$.

tion of $43 \%$ followed by the coordination of 7 with $25 \%$. The other less significant coordination numbers are 8 (13\%), 9 (11 \%), $10(5 \%)$, and $11(3 \%)$. The abundance of chloride ions present in the $\mathrm{K}^{+}$first hydration shell is significantly larger than for $\mathrm{Li}^{+}$ or $\mathrm{Na}^{+}$. In fact, for each coordination number the sum of configurations with $\mathrm{Cl}^{-}$is larger than those with water only. For the most abundant hexa-coordination the relative abundance for the $\mathrm{K}^{+}\left(\mathrm{H}_{2} \mathrm{O}\right)_{5} \mathrm{Cl}^{-}(22 \%) \mathrm{K}^{+}\left(\mathrm{H}_{2} \mathrm{O}\right)_{4} \mathrm{Cl}_{2}^{-}$ $(6 \%)$ is larger than $\mathrm{K}^{+}\left(\mathrm{H}_{2} \mathrm{O}\right)_{6}(14 \%)$. For the hepta- and octa-coordinations, the abundance of the configuration $\mathrm{K}^{+}\left(\mathrm{H}_{2} \mathrm{O}\right)_{6} \mathrm{Cl}^{-}(9 \%)$ vs $\mathrm{K}^{+}\left(\mathrm{H}_{2} \mathrm{O}\right)_{7}(11 \%)$, and $\mathrm{K}^{+}\left(\mathrm{H}_{2} \mathrm{O}\right)_{7} \mathrm{Cl}^{-}(6 \%)$ vs $\mathrm{K}^{+}\left(\mathrm{H}_{2} \mathrm{O}\right)_{8}(6 \%)$ are similar. The structures $\mathrm{K}^{+}\left(\mathrm{H}_{2} \mathrm{O}\right)_{6}$ and $\mathrm{K}^{+}\left(\mathrm{H}_{2} \mathrm{O}\right)_{5} \mathrm{Cl}^{-}$are depicted in Figures $6 \mathrm{~A}$ and $6 \mathrm{~B}$. Other structures with abundances higher than $5 \%$ are shown in Figure S4 in SI. 
Water molecules in the first shell of $\mathrm{K}^{+}$are found to be more delocalized than for $\mathrm{Li}^{+}$and $\mathrm{Na}^{+}$. All these observations confirm that the first hydration shell of the $\mathrm{K}^{+}$is the most disordered, which is further supported by the very low probability of voids between the first and second solvation shells (Figures $6 \mathrm{C}$ and $6 \mathrm{D}$ ).

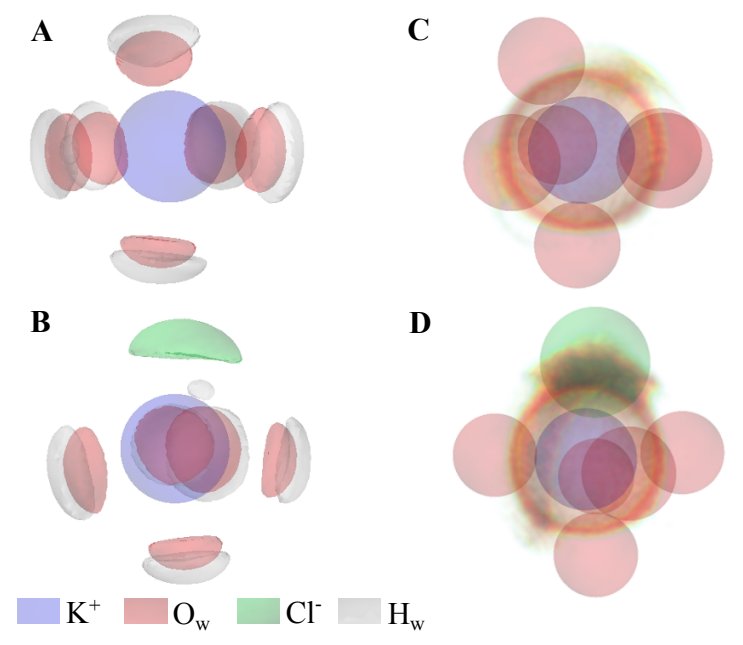

Figure 6: Structures of the two most populated configurations of the first coordination shell for $\mathrm{K}^{+}$. The left column shows density plots of waters or chloride molecules around a central $\mathrm{K}^{+}$. The right column depicts the voids in the same structures, with spheres showing the positions of atoms for reference. The more intense the color, the lower probability of finding oxygen atoms in corresponding region. Panels $\mathbf{A}$ and $\mathbf{C}$ correspond to $\mathrm{K}^{+}\left(\mathrm{H}_{2} \mathrm{O}\right)_{6}$. Panels $\mathbf{B}$ and $\mathbf{D}$ are respective to $\mathrm{K}^{+}\left(\mathrm{H}_{2} \mathrm{O}\right)_{5} \mathrm{Cl}^{-}$.

\section{Comparison of microscopic structures}

The density maps of oxygen, hydrogen, and chloride for configurations displayed in Figures 4, 5, and 6 demonstrate, in agreement with chemical intuition and previous observations, that the disorder of water molecules (as well as that of the chloride ion) coordinated to the central cation increases in the order $\mathrm{Li}^{+}$ $<\mathrm{Na}^{+}<\mathrm{K}^{+}$. In the $\mathrm{Li}^{+}$tetrahedral coordination structure, the distributions of oxygens (and chloride) are highly localized at the four vertices of the tetrahedron accompanied with well-defined "doughnut" clouds in the outer region of the first shell corresponding to hydrogen atoms. This confirms a strong interaction between $\mathrm{Li}^{+}$ion and the first shell of waters leading to a very rigid structure. In comparison to $\mathrm{Li}^{+}$, the water oxygens, as well as hydrogens, surrounding $\mathrm{Na}^{+}$and $\mathrm{K}^{+}$ions show a more diffuse structures.

The observation of a decreasing degree of structural order with increasing cation size is further supported by calculated distributions of angle $\phi$ (i.e., $\mathrm{X}$-cation- $\mathrm{X}$ angle, where $\mathrm{X}=\mathrm{O}$, $\mathrm{Cl}$ ) and the angle $\theta$ (i.e., the angle between the cation-oxygen and water dipole vectors) for the most populated coordination numbers around $\mathrm{Li}^{+}, \mathrm{Na}^{+}$, and $\mathrm{K}^{+}$, see Figure 7 .

For the $\mathrm{Li}^{+}$ion, the distribution of $\phi$ shows a nearly perfect tetrahedral geometry of the first hydration shell, see Fig. 7A). Both O-Li-O, O$\mathrm{Li}-\mathrm{Cl}$ angle distributions around $\mathrm{Li}^{+}$ion for the 4:0 and 3:1 configurations have a single relatively sharp peak around $108^{\circ}$. This value is very close to the $109.5^{\circ}$ of a perfect tetrahedron. Additionally, the complementary $\theta$ angle has an average value of $\sim 180^{\circ}$ and shows the sharpest profile among the studied cations, see Fig. 7B. This large value of the $\theta$ angle suggest that the waters around are strongly bound to the cation, with the oxygen near the cation and hydrogen pointing outwards most of the times.

For $\mathrm{Na}^{+}$the angle distributions of the surrounding waters for 5:0 vs 4:1 configurations are also similar to each other, see Figure 7 . The $\mathrm{X}-\mathrm{Na}^{+}-\mathrm{X} \phi$ angle distribution peaks mainly at $90^{\circ}$ and at $180^{\circ}$, approximately. The $\theta$ distributions exhibits a broader peak at around $180^{\circ}$. These results confirm that the water molecules and chloride ion are placed around $\mathrm{Na}^{+}$in a distorted square pyramid (Fig. 5).

For $\mathrm{K}^{+}$, the distribution of the $\phi$ angle $\left(\mathrm{X}-\mathrm{K}^{+}{ }_{-}\right.$ $\mathrm{X})$ is significantly broader then for the other two cations and it does not exhibit any predominant geometric orientation. The angle between the $\mathrm{K}-\mathrm{O}$ vector and the water molecule dipole vector peaks at around $140^{\circ}$ which is significantly smaller value than for $\mathrm{Na}^{+}$and $\mathrm{Li}^{+}$ $\left(180^{\circ}\right)$, which is in line with a weaker interaction of water molecules with the $\mathrm{K}^{+}$ion.

In conclusion, $\mathrm{Na}^{+}$and $\mathrm{K}^{+}$show broader distributions than $\mathrm{Li}^{+}$meaning that they are described by less defined structures resulting in 

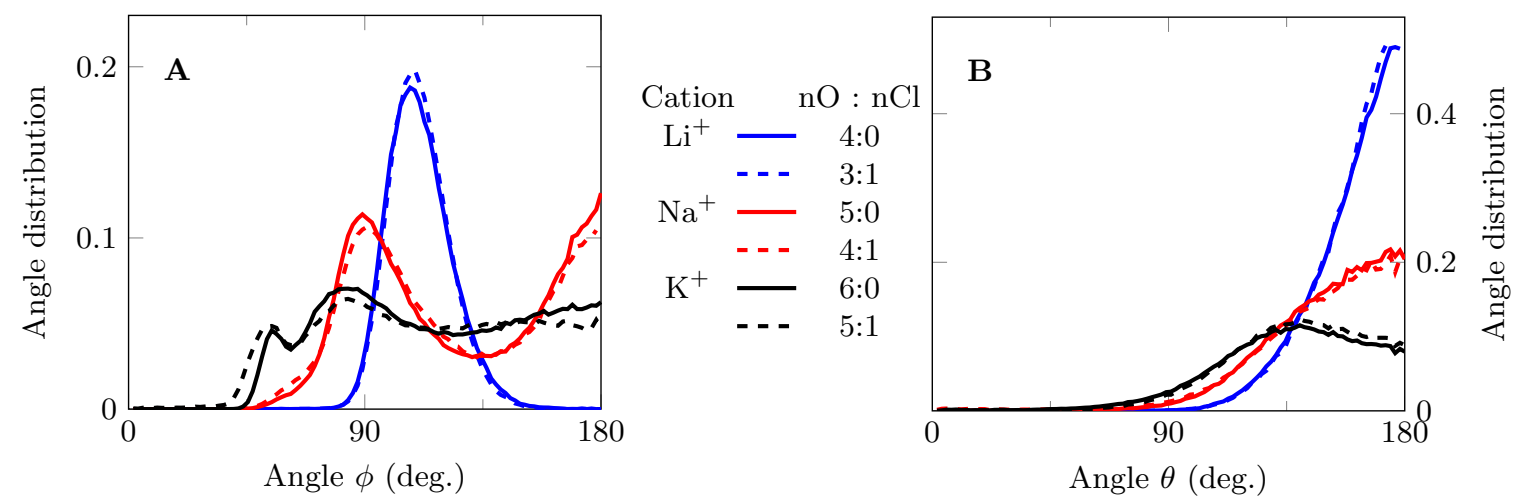

Figure 7: A: Distribution of the $\phi$ angles defined as $X$-cation- $X$ angle where $X$ stands either for water oxygens or $\mathrm{Cl}^{-}$ions. B: Distribution of $\theta$ angles defined between the cation-oxygen and water molecule dipole vectors. Both angles take into account only waters $\mathrm{or}^{-}{ }^{-}$in the first coordination shell of the central cation in a given configuration $(\mathrm{nO}: \mathrm{nCl})$.

a more disordered structure. Additionally, the average angle $\theta$ decreases in the order of $\mathrm{Li}^{+}$ $>\mathrm{Na}^{+}>\mathrm{K}^{+}$. This implies that the first shell water molecules are held tightly around $\mathrm{Li}^{+}$by strong electrostatic forces so that the freedom of rotation of these water molecules is more limited to a single axis, while the larger ions $\mathrm{Na}^{+}$and $\mathrm{K}^{+}$ions exhibit weaker and less constraining interactions with surrounding water molecules in the first shell.

\section{Water oxygen density distribu- tions}

To further compare the arrangement of water molecules in the $\mathrm{LiCl}, \mathrm{NaCl}$, and $\mathrm{KCl}$ solutions, we calculated the three dimensional spatial distribution of oxygen atom densities at a given distance from the central reference cation up to $10 \AA$. We use the radially and density decomposed distribution (r3d) to resolve the average microscopic structures of the local solvent neighborhoods of the studied cations, where the density anomaly between $\mathrm{Na}^{+}$and $\mathrm{Li}^{+}$solutions originates from.

Figures $8, \mathrm{~S} 5$, and $\mathrm{S} 6$ provide the $\mathrm{r} 3 \mathrm{~d}(r, \varrho)$ for each cation and a one to one comparison between each pair, i.e., $\mathrm{Li}^{+}-\mathrm{Na}^{+}, \mathrm{Li}^{+}-\mathrm{K}^{+}$, and $\mathrm{Na}^{+}-\mathrm{K}^{+}$. The presented cation-water oxygen r3d are averaged over all accessible configurations taking into account relative abundances of the coordination numbers as in Figure 3.

Focusing first in the r3d itself (A and B pan- els), we see that the key to understanding the difference in the microscopic structure of $\mathrm{LiCl}$, $\mathrm{NaCl}$, and $\mathrm{KCl}$ solutions are the "voids", i.e., regions in the aligned neighborhood of cations with a low occupational probability or, in terms of the r3d, regions with low density values $\varrho$. Voids are mostly present in the low-distance region up to $4 \AA$. Four distinct regions appear with different functional meanings. The first region is the space occupied by the cation itself. For the relatively small $\mathrm{Li}^{+}$ion the region spans up to $1.8 \AA$, while for the larger $\mathrm{Na}^{+}$ and $\mathrm{K}^{+}$ions it spans further, up to $2.0 \AA$ and $2.3 \AA$, respectively. These results match those obtained for the RDFs. After this first exclusion zone the interpretative power of r3d becomes evident. Another region of voids appears due to the rigid structure of the first solvation shell. Moving from $\mathrm{Li}^{+}(1.8-2.4 \AA)$, via $\mathrm{Na}^{+}$ (2.1-3.0 $\AA)$, to $\mathrm{K}^{+}(2.5-3.5 \AA)$ this shell looses its rigidity resulting in less voids. The third distinct region corresponds to the empty space between the first and second solvation shells. This space is partially occupied by $\mathrm{Cl}^{-}$and water hydrogens. Finally, within the second solvation shell other regions with less voids appear, also due to the shell rigidity. In overall, the variance of density at a given $r$ decreases in the series $\mathrm{Li}^{+}>\mathrm{Na}^{+}>\mathrm{K}^{+}$denoting decreasing ordering within the cation series. However, this decrease on variance does not occur for all radial distances.

To quantify better the differences between 

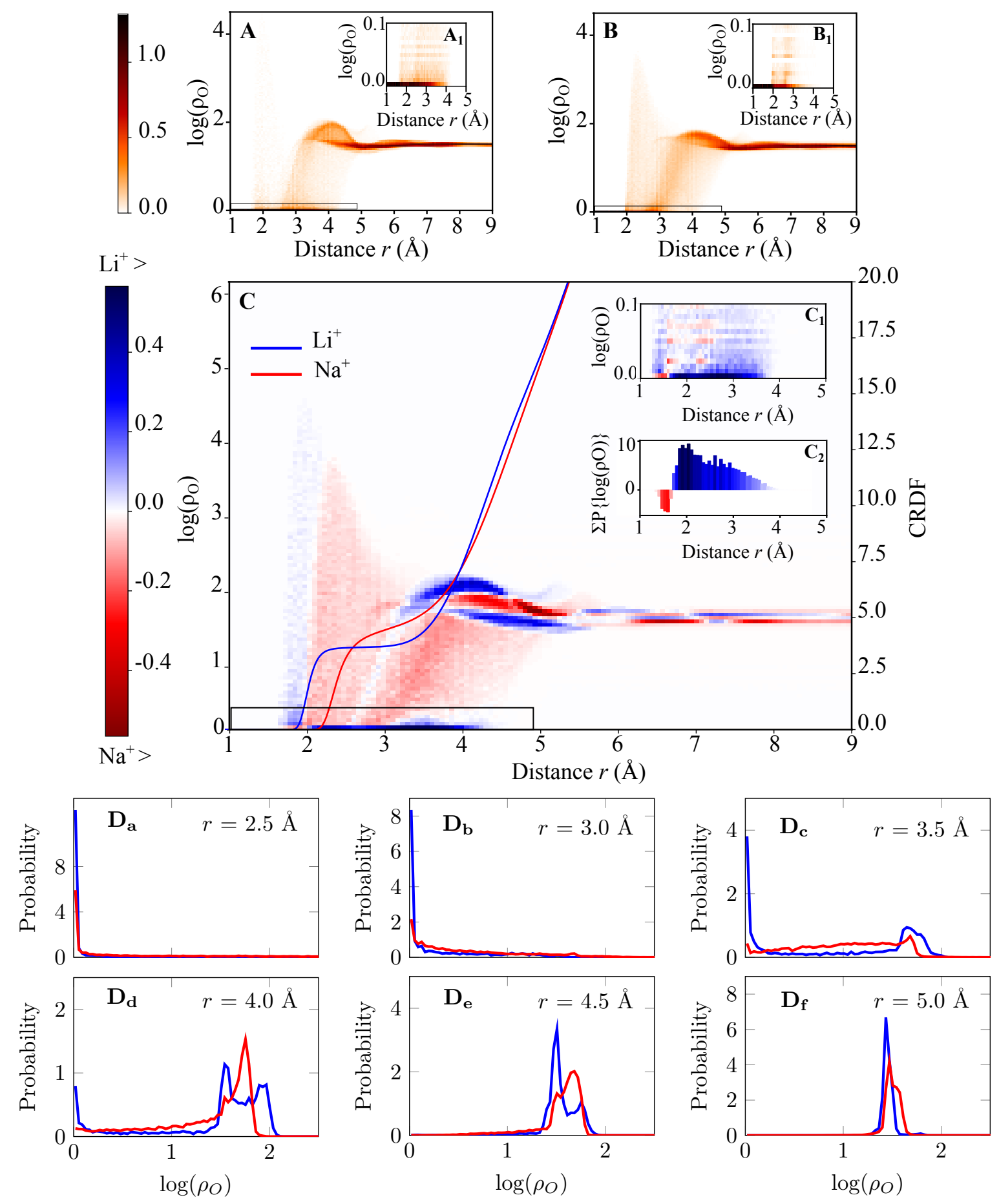

Figure 8: A, B: The radially and density decomposed distributions (r3d) of water oxygen atom around the $\mathrm{Li}^{+} \mathrm{r} 3 \mathrm{~d}_{\mathrm{Li}}(r, \varrho)$ and $\mathrm{Na}^{+}$ions $\mathrm{r}_{\mathrm{N}} \mathrm{Na}(r, \varrho)$. C: The function represents the difference between functions $\mathrm{A}\left(\mathrm{Li}^{+}\right)$and function $\left.\mathrm{B}\right)\left(\mathrm{Na}^{+}\right), \Delta \mathrm{r} 3 \mathrm{~d}_{\mathrm{Li}-\mathrm{Na}}$. The most significant domains wrapped by rectangle in figure $\mathrm{C}$ are plotted in $\mathrm{C}_{1}$, where $\mathrm{C}_{1}$ is the $\Delta \mathrm{r} 3 \mathrm{~d}_{\mathrm{Li}-\mathrm{Na}}(r, \varrho)$ in terms of void region of density. $\mathrm{C}_{2}$ : the total number of $\Delta \mathrm{r} 3 \mathrm{~d}_{\mathrm{Li}-\mathrm{Na}}$ counted with respect to given distance. $\mathrm{D}$ : Representative cuts of the r3d functions calculated at selected radii from the central cations. 
cations, we can subtract the r3d of one cation from another. Figure $8 \mathrm{C}$ shows the difference

$$
\Delta \mathrm{r} 3 \mathrm{~d}_{\mathrm{Li}-\mathrm{Na}}=\mathrm{r} 3 \mathrm{~d}_{\mathrm{Li}}(r, \varrho)-\mathrm{r} 3 \mathrm{~d}_{\mathrm{Na}}(r, \varrho)
$$

where $\mathrm{r} 3 \mathrm{~d}_{\mathrm{Li}}(r, \varrho)$ and $\mathrm{r} 3 \mathrm{~d}_{\mathrm{Na}}(r, \varrho)$ are shown in Figure $8 \mathrm{~A}, \mathrm{~B}$. The value of $\Delta \mathrm{r} 3 \mathrm{~d}$ is represented by a color scale. If at a given radial distance $r$ the probability to find an oxygen number density $\varrho$ is higher for $\mathrm{Li}^{+}$than for $\mathrm{Na}^{+}$, then $\Delta \mathrm{r} 3 \mathrm{~d}_{\mathrm{Li}-\mathrm{Na}}$ is positive and the corresponding region in Figure 8 is colored blue. In the opposite case, it is colored red. Analogous plots for the $\mathrm{Li}^{+}-\mathrm{K}^{+}$and $\mathrm{Na}^{+}-\mathrm{K}^{+}$pairs are presented in Figures S5C and S6C in the SI. In each case the smaller cation has an earlier onset of the number density due to is closer first coordination shell, which is shown as an initial higher blue band. This band is followed by a shorter but wider red band where the larger cation reduces the number density difference from the smaller cation. After that point a more complex zone appears with alternate colors, although a clear upper blue narrow wedge arises $(3-5 \AA)$. Relative changes in this zone are harder to rationalize and quantify, as they need a weighted average and are only apparent when overlaid to the correspondent CRDF.

An easier way to rationalize the $\mathrm{Li}^{+}-\mathrm{Na}^{+}$density anomaly is to focus on the voids, which provide a complementary and more concentrated information. The difference in the total volume of voids between $\mathrm{Li}^{+}$and $\mathrm{Na}^{+}$is magnified in Figure $8 \mathrm{C}_{1}$ with its integrated form presented in Figure $8 \mathrm{C}_{2}$. The amount of voids is considerably larger for $\mathrm{Li}^{+}$than for $\mathrm{Na}^{+}$(and also for $\mathrm{K}^{+}$, see Figure S5C in the SI), except at low $r$ as $\mathrm{Li}^{+}$is the smallest cation. The occurrence of voids in the aligned neighborhood signifies lower flexibility of the hydration shells, hence the reduced packing capability. Consequently, the average number density of the $\mathrm{LiCl}$ solution is lowered compared to what one would intuitively assume, becoming comparable to that of $\mathrm{NaCl}$.

The rigidity of the solvation shell of $\mathrm{Li}^{+}$results not only in the increased occurrence of voids, but also in an increased variance of the r3d at a given $r$. Representative cuts for dis- tinct regions (between 2.5 and $5 \AA$ ) are presented in the Figures $8 \mathrm{D}_{\mathrm{a}-\mathrm{f}}$. For $\mathrm{Li}^{+}$, the distributions at the distances $r \approx 3.5$ to $4.0 \AA$ are multi-modal (have two or more distinct peaks) and, thus, exhibit larger variance than the mostly uni-modal distributions found for $\mathrm{Na}^{+}$. This shows that also the second hydration shell of $\mathrm{Li}^{+}$is more rigid and more structured compared to $\mathrm{Na}^{+}$.

Figure 8 further demonstrates the two interplaying causes of the density anomaly between $\mathrm{Li}^{+}$and $\mathrm{Na}^{+}$solutions. First, there are significantly more voids in case of the $\mathrm{Li}^{+}$than $\mathrm{Na}^{+}$. Second, the solvation shell of $\mathrm{Li}^{+}$is more constrained and rigid. Together, these features make the solvent neighborhoods of $\mathrm{Li}^{+}$ less packed than naively expected, thus decreasing the average number density. Nevertheless, it is a pure coincidence that at ambient conditions these effects lower the bulk oxygen number density of the aqueous $\mathrm{LiCl}$ solution such that it practically coincides with that of $\mathrm{NaCl}$.

\section{Hydrogen bonding network}

The lack of flexibility of the first hydration shell around $\mathrm{Li}^{+}$results in heavily constrained water molecules. This in turn results in less densely pack solvent neighborhood. The presence of heavily constrained water molecules around $\mathrm{Li}^{+}$can be quantified by characterizing its hydrogen bond network, which we then compare to those in $\mathrm{Na}^{+}$and $\mathrm{K}^{+}$. Figure 9 shows the average number of hydrogen bonds per water molecule $\left(n_{H B}^{w}\right)$ in the first coordination shells of $\mathrm{Li}^{+}, \mathrm{Na}^{+}$, and $\mathrm{K}^{+}$. A hydrogen bond was defined using the set of geometrical criteria, i.e., the $\mathrm{O} \cdots \mathrm{O}$ distance is shorter than $3.5 \AA$ and the $\mathrm{O}-\mathrm{H} \cdots \mathrm{O}$ angle lower than $145^{\circ} 46$. We see a mild decrease in the number of hydrogen bonds with decreasing the size of the cation. Also, for all three solutions these numbers are significantly lower than in pure water.

Table S1 breaks down the hydrogen bonds contributions in the first coordination shell for each major configuration (weight over $5 \%$ ). The hydrogen bond calculation is separated into those between water molecules of the first hydration shell, and those between the first hy- 
dration shell and the second one. The latter is also decomposed into $\mathrm{H}$-bond acceptor and donor contributions. For both hydration shells the total number of hydrogen bonds per water molecule in the first shell increases with the size of the cation regardless of the hydration coordination. The low flexibility of water molecules bound to $\mathrm{Li}^{+}$is due to strong cationwater electrostatic forces compromising waterwater hydrogen bonding. In contrast, the larger ions, $\mathrm{Na}^{+}$and $\mathrm{K}^{+}$, with lower charge densities have weaker interactions with surrounding water molecules in the first shell. This allows these water molecules to orient more freely in such a way that they can form more hydrogen bonds to each other.

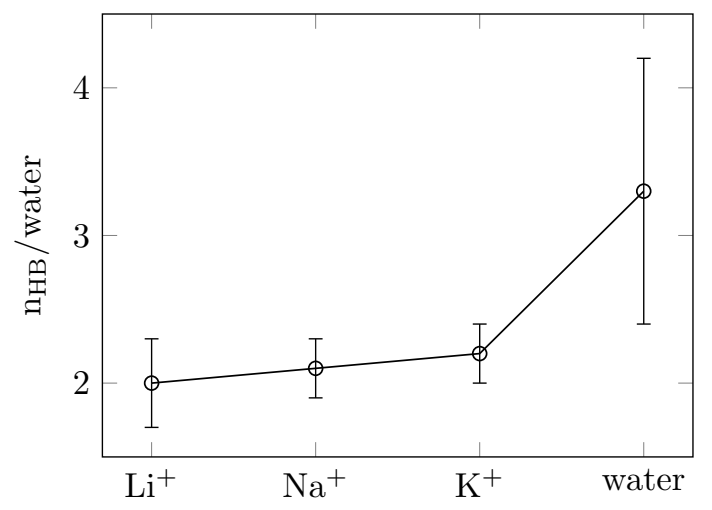

Figure 9: Average number and standard deviation of hydrogen bond per water molecule in the first shell calculated for all hydration configurations with contributions higher than $5 \%$.

\section{Conclusion}

In this study, we report on detailed interpretation of experimental findings concerning structures and number densities of concentrated aqueous solutions of alkali chlorides based on molecular dynamics simulations. In good agreement with previous measurements, the calculated number densities are 0.0971, 0.0965 and 0.09716 atoms $/ \AA$ for $4 \mathrm{~m} \mathrm{LiCl}$, $\mathrm{NaCl}$, and $\mathrm{KCl}$ solutions, respectively. Next, we provide molecular insight into the counterintuitive fact that the number density values for $\mathrm{Na}^{+}$and $\mathrm{Li}^{+}$solutions are practically equal to each other. Detailed analysis of molecu- lar simulations shows that water molecules are less well packed around $\mathrm{Li}^{+}$than around the larger studied cations, $\mathrm{Na}^{+}$and $\mathrm{K}^{+}$, due to the relatively higher rigidity of the $\mathrm{Li}^{+}$solvation shells and, consequently, due to the increased presence of voids - on average empty regions of space - in the aligned neighborhood of the cation. Additionally, the tightly constrained water molecules in the first shell around $\mathrm{Li}^{+}$ cannot engage as easily in hydrogen bonding. Put all together, this provides a molecular interpretation of the matching number densities of concentrated $\mathrm{LiCl}$ and $\mathrm{NaCl}$ aqueous solutions at ambient conditions.

\section{Associated Content}

\section{Supporting information}

The Supporting Information is available free of charge on the ACS Publications website.

Acknowledgement OT and MNTH acknowledge support from the Faculty of Mathematics and Physics of the Charles University where they are enrolled as PhD students and from the International Max Planck Research School for "Many-Particle Systems in Structured Environments" (Dresden, Germany) for support. Computational resources were supplied by the project "e-Infrastruktura CZ" (eINFRA LM2018140) provided within the program Projects of Large Research, Development and Innovations Infrastructures. PJ acknowledges the Czech Science Foundation for support via an EXPRO grant no. 19-26854X. HMS acknowledge the European Regional Development Fund OP RDE (project ChemBioDrug no. CZ.02.1.01/0.0/0.0/16_019/0000729) for support and computational resources. 


\section{References}

(1) Marcus, Y. Ions in Solution and their Solvation; John Wiley \& Sons, Inc., 2015.

(2) Wolfgang, V. Chemistry of Salts in Aqueous Solutions: Applications, Experiments, and Theory. Pure and Applied Chemistry 2011, 83, 1015-1030.

(3) Marcus, Y. Effect of Ions on the Structure of Water: Structure Making and Breaking. Chemical Reviews 2009, 109, 1346-1370.

(4) Bouazizi, S.; Nasr, S. Structural investigations of high concentrated aqueous $\mathrm{LiCl}$ solutions: X-ray scattering and MD simulations approach. Journal of Molecular Structure 2008, 875, 121-129.

(5) Iradwikanari, W.; Congcong, H.; Dennis, N.; Uwe, B.; Thomas, M. W.; Lars, G. M. P.; Nilsson, A. The structure of water in the hydration shell of cations from $\mathrm{x}$ ray Raman and small angle x-ray scattering measurements. The Journal of chemical physics 2011, 134, 064513.

(6) Antalek, M.; Pace, E.; Hedman, B.; Hodgson, K. O.; Chillemi, G.; Benfatto, M.; Sarangi, R.; Frank, P. Solvation structure of the halides from x-ray absorption spectroscopy. The Journal of Chemical Physics 2016, 145, 044318.

(7) Nagasaka, M.; Yuzawa, H.; Kosugi, N. Interaction between Water and Alkali Metal Ions and Its Temperature Dependence Revealed by Oxygen K-Edge X-ray Absorption Spectroscopy. The Journal of Physical Chemistry B 2017, 121, 10957-10964.

(8) Kameda, Y.; Maeda, S.; Amo, Y.; Usuki, T.; Ikeda, K.; Otomo, T. Neutron Diffraction Study on the Structure of Hydrated $\mathrm{Li}^{+}$in Dilute Aqueous Solutions. The Journal of Physical Chemistry B 2018, 122, 1695-1701.

(9) Mason, P. E.; Ansell, S.; Neilson, G. W.; Rempe, S. B. Neutron Scattering Studies of the Hydration Structure of $\mathrm{Li}^{+}$. The
Journal of Physical Chemistry B 2015, 119, 2003-2009.

(10) Schwaab, G.; Sebastiani, F.; Havenith, M. Ion Hydration and Ion Pairing as Probed by $\mathrm{THz}$ Spectroscopy Angewandte Reviews. Angewandte Chemie International Edition 2019, 58, 3000-3013.

(11) Alex, P. G.; Cui, Z.; François, G.; Giulia, G. Structural and electronic properties of aqueous $\mathrm{NaCl}$ solutions from ab initio molecular dynamics simulations with hybrid density functionals. Chemical Physics Letters 2014, 604, 89-96.

(12) Jalilehvand, F.; Spångberg, D.; LindqvistReis, P.; Hermansson, K.; Persson, I.; Sandström, M. Hydration of the Calcium Ion. An EXAFS, Large-Angle X-ray Scattering, and Molecular Dynamics Simulation Study. Journal of the American Chemical Society 2001, 123, 431-441.

(13) Mehandzhiyski, A. Y.; Riccardi, E.; Erp, T. S. V.; Trinh, T. T.; Grimes, B. A. Ab Initio Molecular Dynamics Study on the Interactions between Carboxylate Ions and Metal Ions in Water. The Journal of Physical Chemistry B 2015, 119, 1071010719.

(14) Sripa, P.; Tongraar, A.; Kerdcharoen, T. "Structure-Making" Ability of $\mathrm{Na}^{+}$in Dilute Aqueous Solution: An ONIOM-XS MD Simulation Study. The Journal of Physical Chemistry A 2013, 117, 18261833.

(15) Payaka, A.; Tongraar, A.; Rode, B. M. QM/MM Dynamics of $\mathrm{CH}_{3} \mathrm{COO}^{-}$- Water Hydrogen Bonds in Aqueous Solution. The Journal of Physical Chemistry A 2010, 114, 10443-10453.

(16) Rempe, S. B.; Pratt, L. R.; Hummer, G.; Kress, J. D.; Martin, R. L.; Redondo, A. The Hydration Number of $\mathrm{Li}^{+}$in Liquid Water. Journal of the American Chemical Society 2000, 122, 966-967. 
(17) Pluhařová, E.; Jungwirth, P.; Matubayasi, N.; Maršálek, O. Structure and Dynamics of the Hydration Shell: Spatially Decomposed Time Correlation Approach. Journal of Chemical Theory and Computation 2019, 15, 803-812.

(18) Mason, P. E.; Neilson, G. W.; Price, D.; Saboungi, M.-L.; Brady, J. W. Simulation and Neutron Diffraction Studies of Small Biomolecules in Water. Food Biophysics. 2011, 6, 210-216.

(19) Kohagen, M.; Mason, P. E.; Jungwirth, P. Accounting for Electronic Polarization Effects in Aqueous Sodium Chloride via Molecular Dynamics Aided by Neutron Scattering. The Journal of Physical Chemistry B 2016, 120, 1454-1460.

(20) Berendsen, H. J. C.; Grigera, J. R.; Straatsma, T. P. The missing term in effective pair potentials. The Journal of Physical Chemistry 1987, 91, 6269-6271.

(21) Mason, P. E.; Tavagnacco, L.; Saboungi, M.-L.; Hansen, T.; Fischer, H. E.; Neilson, G. W.; Ichiye, T.; Brady, J. W. Molecular Dynamics and Neutron Scattering Studies of Potassium Chloride in Aqueous Solution. The Journal of Physical Chemistry B 2019, 123, 10807-10813.

(22) Kohagen, M.; Mason, P. E.; Jungwirth, P. Accurate Description of Calcium Solvation in Concentrated Aqueous Solutions. The Journal of Physical Chemistry $B$ 2014, 118, 7902-7909.

(23) Igor, L.; Alexei, S. Accounting for electronic polarization in non-polarizable force fields. Physical Chemistry Chemical Physics 2011, 13, 2613-2626.

(24) Duboué-Dijon, E.; Javanainen, M.; Delcroix, P.; Jungwirth, P.; MartinezSeara, H. A practical guide to biologically relevant molecular simulations with charge scaling for electronic polarization.
The Journal of Chemical Physics 2020, 153, 050901.

(25) Mason, P. E.; Wernersson, E.; Jungwirth, P. Accurate Description of Aqueous Carbonate Ions: An Effective Polarization Model Verified by Neutron Scattering. The Journal of Physical Chemistry B 2012, 116, 8145-8153.

(26) Shannon, R. D. Revised effective ionic radii and systematic studies of interatomic distances in halides and chalcogenides. Acta Crystallographica Section A 1976, 32, 751-767.

(27) Essmann, U.; Perera, L.; Berkowitz, M. L.; Darden, T.; Lee, H.; Pedersen, L. G. A smooth particle mesh Ewald method. The Journal of Chemical Physics 1995, 103, 8577-8593.

(28) Hoover, W. G. Canonical dynamics: Equilibrium phase-space distributions. Physical Review A 1985, 31, 1695-1697.

(29) Nosé, S. A molecular dynamics method for simulations in the canonical ensemble. Molecular Physics 1984, 52, 255-268.

(30) Parrinello, M.; Rahman, A. Polymorphic transitions in single crystals: A new molecular dynamics method. Journal of Applied Physics 1981, 52, 7182-7190.

(31) Miyamoto, S.; Kollman, P. A. Settle: An analytical version of the SHAKE and RATTLE algorithm for rigid water models. Journal of Computational Chemistry 1992, 13, 952-962.

(32) Abraham, M. J.; Murtola, T.; Schulz, R.; Páll, S.; Smith, J. C.; Hess, B.; Lindahl, E. GROMACS: High performance molecular simulations through multi-level parallelism from laptops to supercomputers. SoftwareX 2015, 1-2, 19-25.

(33) Ramachandran, P.; Varoquaux, G. Mayavi: 3D Visualization of Scientific Data. Computing in Science $\&$ Engineering 2011, 13, 40-51. 
(34) Yamanaka, K.; Yamagami, M.; Takamuku, T.; Yamaguchi, T.; Wakita, H. Xray diffraction study on aqueous lithium chloride solution in the temperature range 138-373 K. The Journal of Physical Chemistry 1993, 97, 10835-10839.

(35) Bouazizi, S.; Nasr, S. Local order in aqueous lithium chloride solutions as studied by X-ray scattering and molecular dynamics simulations. Journal of Molecular Structure 2007, 837, 206-213.

(36) Cavallari, M.; Cavazzoni, C.; Ferrario, M. Structure of $\mathrm{NaCl}$ and $\mathrm{KCl}$ concentrated aqueous solutions by ab initio molecular dynamics. Molecular Physics 2004, 102, 959-966.

(37) Neilson, G. W.; Skipper, N. K ${ }^{+}$coordination in aqueous solution. Chemical Physics Letters 1985, 114, 35-38.

(38) Ohtomo, N.; Arakawa, K. Neutron Diffraction Study of Aqueous Ionic Solutions. II. Aqueous Solutions of Sodium Chloride and Potassium Chloride. Bulletin of the Chemical Society of Japan 1980, 53, 1789-1794.

(39) Rudolph, W.; Brooker, M. H.; Pye, C. C. Hydration of Lithium Ion in Aqueous Solutions. The Journal of Physical Chemistry 1995, 99, 3793-3797.

(40) Varma, S.; Rempe, S. B. Coordination Numbers of Alkali Metal Ions in Aqueous solutions. Biophysical Chemistry 2006, 124, 192-199.

(41) Ansell, S.; Barnes, A. C.; Mason, P. E.; Neilson, G. W.; Ramos, S. X-ray and Neutron Scattering Studies of the Hydration Structure of Alkali Ions in Concentrated Aqueous Solutions. Biophysical Chemistry 2006, 124, 171-179.

(42) Soper, A. K.; Weckström, K. Ion solvation and water structure in potassium halide aqueous solutions. Biophysical Chemistry 2006, 124, 180-191.
(43) Loeffler, H. H.; Rode, B. M. The hydration structure of the Lithium ion. The Journal of Chemical Physics 2002, 117, 110-117.

(44) Lyubartsev, A. P.; Laasonen, K.; Laaksonen, A. Hydration of $\mathrm{Li}^{+}$ion. An ab initio Molecular Dynamics Simulation. The Journal of Chemical Physics 2001, 114, 3120-3126.

(45) Petit, L.; Vuilleumier, R.; Maldivi, P.; Adamo, C. Ab Initio Molecular Dynamics Study of a Highly Concentrated LiCl Aqueous Solution. Journal of Chemical Theory and Computation 2008, 4, 10401048 .

(46) Guàrdia, E.; Martí, J.; Garcí-Tarrés, L.; Laria, D. A molecular dynamics simulation study of hydrogen bonding in aqueous ionic solutions. Journal of Molecular Liquids 2005, 117, 63-67. 